\title{
Giant Serpentine Internal Carotid Artery Aneurysm with Complex Aortic and Supra-aortic Vascular Anatomy: Endovascular Treatment through Direct Percutaneous Carotid Puncture
}

\author{
Sibasankar Dalai ${ }^{1} \quad$ Uday Limaye $^{2} \quad$ R. V. Narayana ${ }^{1} \quad$ Guru Prasad $^{2} \quad$ Rajesh Pati $^{1} \quad$ Sunanda Anand ${ }^{2}$ \\ ${ }^{1}$ Seven Hills Hospital, Rockdale Layout, Visakhapatnam, \\ Andhra Pradesh, India \\ 2Leelavathi Hospital, Bandra West, Mumbai, Maharashtra, India \\ Address for correspondence Sibasankar Dalai, MD, SevenHills Hospital, \\ 11-4-4/A, Waltair Main Road Ram Nagar, Rockdale Layout, Visakhapatnam, \\ Andhra Pradesh 530002, India (e-mail: sibasankar@gmail.com).
}

Indian J Neurosurg 2019;8:58-62

\begin{abstract}
Keywords

- serpentine aneurysm

- embolization

- direct carotid puncture

Giant serpentine aneurysm (GSA) with rare aortic and supra-aortic vascular anomaly is technically difficult to treat. Often such patients suffer without treatment. We have treated a patient by a different approach which is not regularly followed. This case has many developmental anomalies with complicated anatomy, and it is a challenge to the treating interventionist.
\end{abstract}

\section{Introduction}

Giant serpentine aneurysms (GSAs) are rare; fusiform aneurysms, were first documented by Segal and McLaurin in 1977 as subcategories of giant aneurysms. ${ }^{1}$ A serpentine aneurysm has been defined as "a giant (greater than $2.5 \mathrm{~cm}$ in diameter), partially thrombosed aneurysm, with tortuous vascular channels that have a separate entrance and outflow pathway."1 GSAs lead to unusual arterial dilations, thereby extending the lumen longitudinally along the axis and curves of the original artery, while creating a serpiginous pathway with a distinct entrance and outflow tract.,., They can be categorized under large intracranial aneurysms, which have distinctive computed tomographic (CT), magnetic resonance imaging (MRI), and angiographic features. These aneurysms can easily be mistaken for neoplasms, if unrecognized. They often present with progressive neurologic deficits, and CT and MR imaging studies often show their association with adjacent edema and mass effect. ${ }^{4}$ GSA can be confirmed through cerebral angiography which can further facilitate the course of its treatment. We describe a case of GSA of right internal carotid artery (ICA) with unusual arch anatomy. As it is difficult to approach the aneurysm from either by femoral or brachial route we had to do a direct percutaneous carotid puncture.

\section{Case Presentation}

A 43-year-old nondiabetic, nonhypertensive woman, with no significant past medical history, came with complaints of severe headache, diminishing vision, and bulging of right eye ball ( - Fig. 1). Clinical examination by an ophthalmologist showed right-sided ptosis, opthalmoplegia, diplopia, and proptosis. Her magnetic resonance imaging (MRI) brain scan with MR angiogram revealed a GSA in the right internal carotid artery (ICA; - Figs. $\mathbf{2}$ and $\mathbf{3}$ ).

A digital subtraction angiogram (DSA) could not be completed due to difficulty in navigating the catheter into the right brachiocephalic artery. Hence, CT angiogram of the aorta, supra-aortic blood vessels, and cerebral arteries was done that revealed a retroesophageal aortic arch with absent left vertebral artery (VA) ( - Fig. 4). CT angiogram also showed hypoplastic left common carotid artery (CCA) with agenesis of left ICA. There was also a giant serpentine right ICA aneurysm that involved the petrous, cavernous, and supraclinoid ICA along with fenestration of the right VA. An additional arch aortogram further demonstrated an arch with left subclavian artery coming out as a major branch from the aorta and the right half of the aortic arch taking a steep round curve to continue as right innominate artery and a right CCA.
DOI https://doi.org/

10.1055/s-0039-3402537

ISSN 2277-954X.
License terms

(ब) (1) $\Theta \circledast$ 


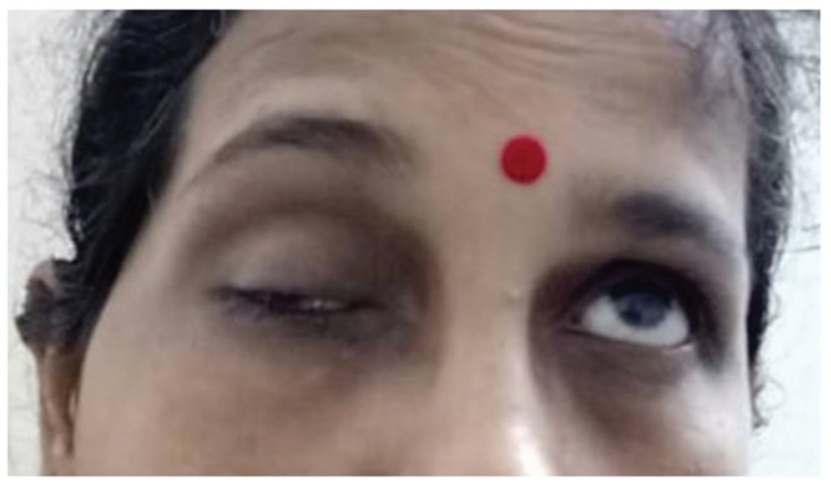

Fig. 1 A 43-year-old female presenting with right acute ptosis, ophthalmoplegia.

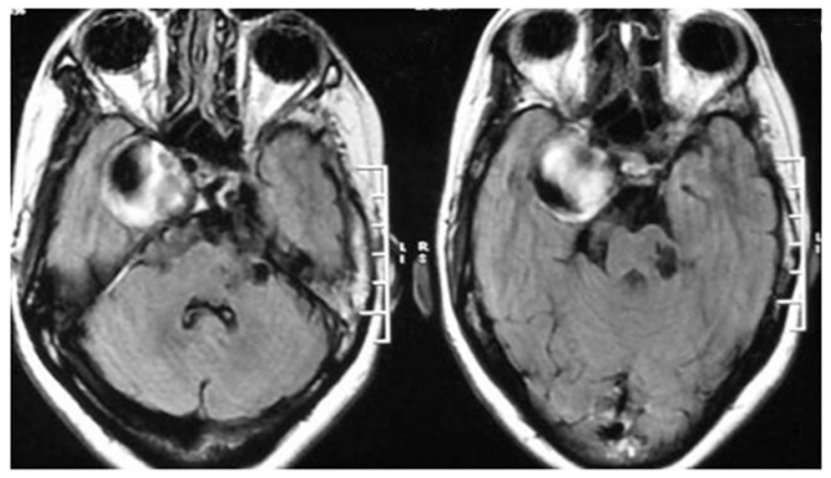

Fig. 2 MRI imaging demonstrating a giant partially thrombosed aneurysm of right ICA. ICA, internal carotid artery; MRI, magnetic resonance imaging.

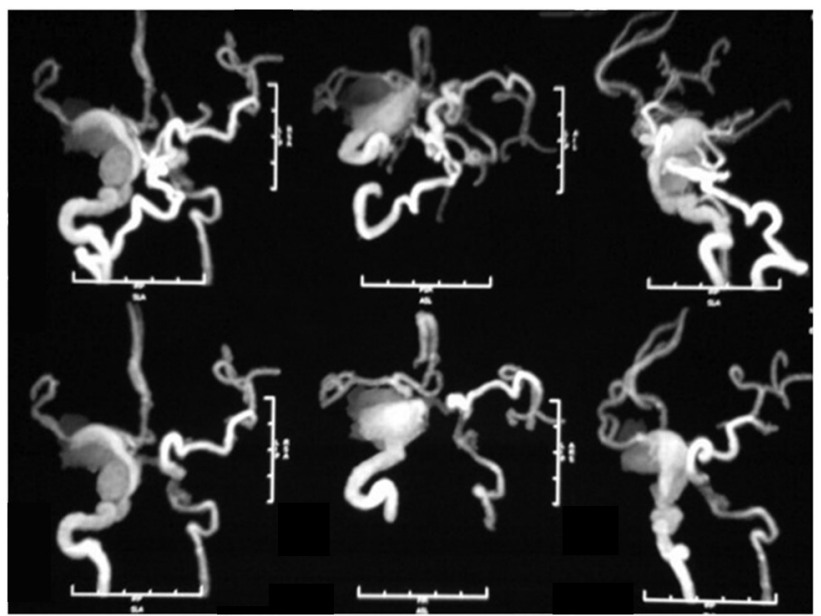

Fig. 3 MRA demonstrating a giant serpentine aneurysm involving the right petrous, cavernous, and supraclinoid ICA. ICA, internal carotid artery; MRA, magnetic resonance angiography.

\section{Treatment Plan}

The patient was taken for endovascular management for GSA of right ICA. We tried to reach the right CCA through right transfemoral arterial route and right brachial artery percutaneous route but were not successful. Failing to cannulate right CCA due to extreme tortuosity, we placed a 5-F head hunter catheter (Cook Medical; Indiana, United States) into the right vertebral artery to perform an angiogram. A direct percutaneous right carotid puncture

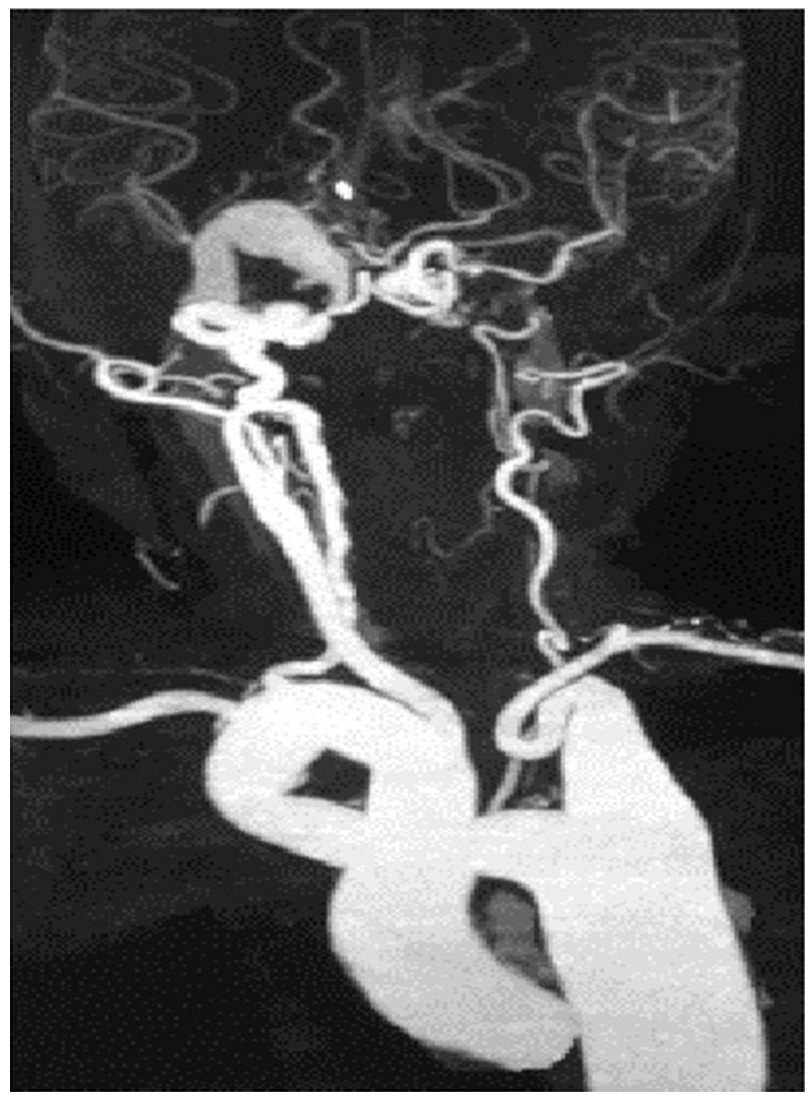

Fig. 4 CT angiogram demonstrating, complex anatomy of the arotic arch and supra-aortic and intracranial blood vessels including giant serpentine right petrocavernous ICA aneurysm, circumflex retroesophageal right aortic arch, small left CCA with left ICA agenesis, vertebral artery fenestration with persistent trigeminal artery. CCA, common carotid artery; ICA, internal carotid artery.

was planned under conscious sedation for access into the GSA. Under ultrasound guidance, with Seldinger technique, a single carotid artery wall puncture was obtained. A 0.035-J tip short wire was advanced under fluoroscopic guidance. The puncture needle was removed and a 6-F short radial sheath (Cordis Inc., Florida, United States) was placed into the right CCA.

Angiogram of the right CCA was performed. Angiogram demonstrated the right GSA in the posterior communicating segment of the right ICA with very sluggish distal flow into the cerebral circulation ( $\mathbf{- F i g . 5 A , 5 B}$ ).

A balloon test occlusion was planned through the right CCA sheath to evaluate the cerebral circulation post-coil embolization of the GSA and the parent vessel. A $4 \mathrm{~mm} \times 20 \mathrm{~mm}$ hyperglide balloon (Medtronic Inc., Minnesota, United States) was navigated into the right carotid sheath and placed into the right ICA (petrous course), proximal to the aneurysm. The balloon was inflated and ICA was occluded, balloon occlusion test was done for $30 \mathrm{~min}$ utes under local anesthesia. No hypotension occurred during the procedure ( $\mathbf{- F i g .} \mathbf{6 A})$. A thorough neurological examination was performed which showed no new neurodeficits. Right vertebral artery angiogram from the right transbrachial catheter was performed. The angiogram 
demonstrated a complete cerebral parenchymogram with

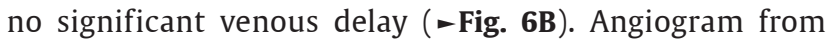
the right CCA sheath demonstrated occluded ICA with the balloon, opacification of right ICA through ophthalmic collaterals.

The right ICA balloon was deflated and removed; the GSA was cannulated with SL 10 microcatheter and Synchro 0.014 wire (Stryker Neurovascular Inc., Michigan, United States). The GSA with the parent artery was embolized by serially deploying detachable platinum coils (Stryker Neurovascular Inc.) till the aneurysm was completely excluded from the circulation with occlusion of the right ICA ( - Fig. 7). Final angiogram demonstrated a well-compensated cerebral parenchymogram in all phases of the angiogram ( $\boldsymbol{- F i g . 8}$ ). Patient tolerated the procedure well. She was treated in the intensive care and subsequently in the ward. Patient was discharged 3 days after the procedure without any new neurodeficits. Antiplatelet drugs, Ecosprin $150 \mathrm{mg}$ (enteric coated Aspirin; acetylsalicylic acid, Leverkusen, Germany) and Plavix $75 \mathrm{mg}$ (clopidogrel; Sanofi-aventis and Bristol-Myers Squibb, United States) were given for first 6 months and later patient is on only Ecosprin 150 mg daily.

Follow-up at 3 months shows improvement in headache, right opthalmoplegia, ptosis, and diplopia (-Fig. 9). Since the patient is under follow-up for the last 2 years without any symptoms or signs of recurrence, repeat angiogram was not done.
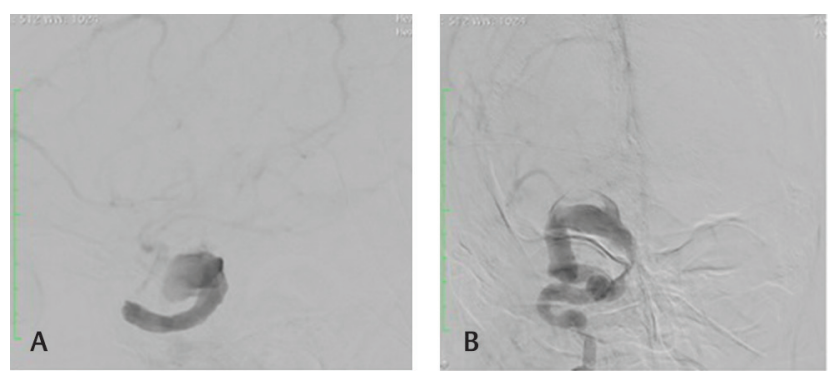

Fig. 5 (A) Lateral view digital subtraction angiogram of right posterior-communicating artery showing the giant serpentine aneurysm. (B) AP view digital subtraction angiogram of the right posterior-communicating-artery giant serpentine aneurysm. AP, anteroposterior.
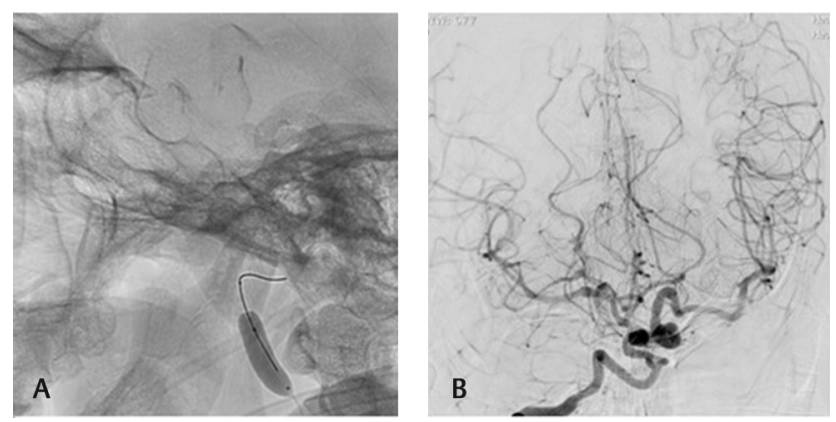

Fig. 6 (A) Balloon Inflated in the right ICA proximal to GSA. (B) Postballoon occlusion test angiogram. GSA, giant serpentine aneurysm; ICA, internal carotid artery.

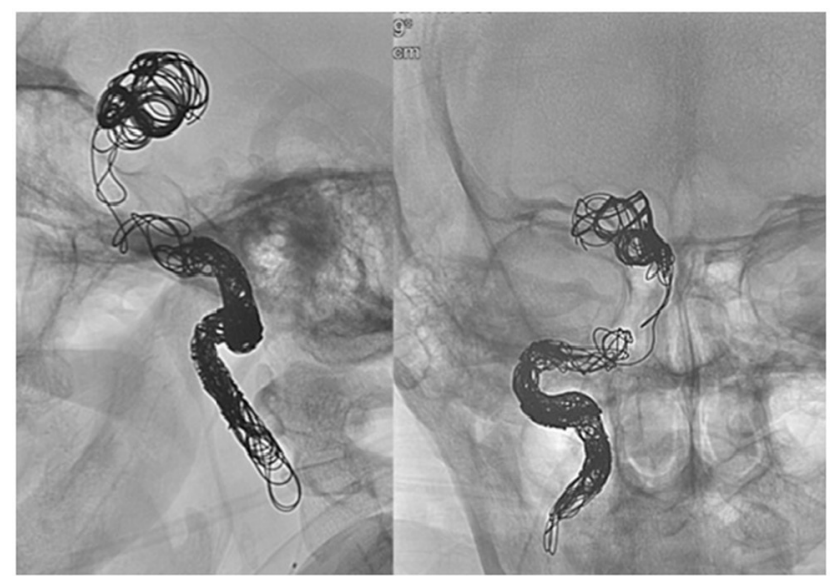

Fig. 7 Coil embolization of the giant serpentine aneurysm with parent artery occlusion.

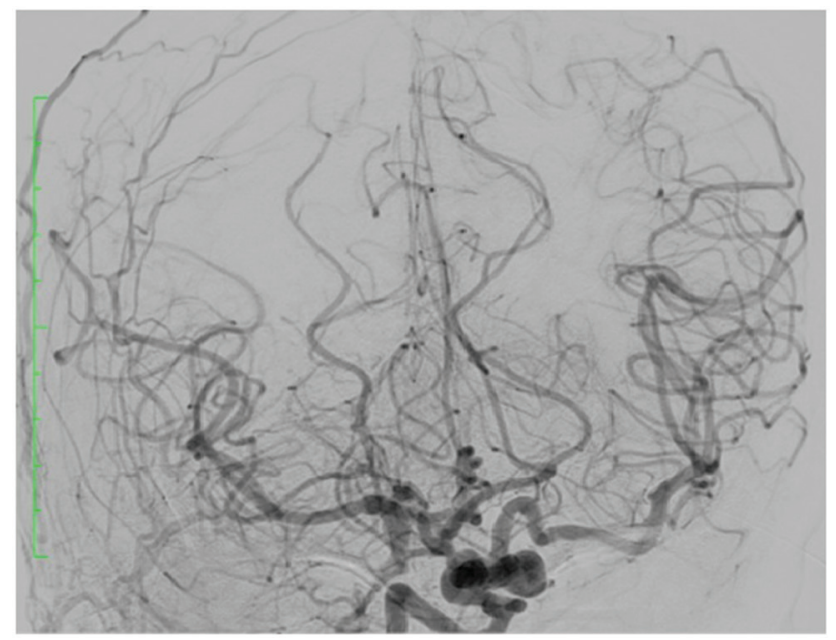

Fig. 8 Postcoiling, final angiogram from the right vertebral artery demonstrating complete cerebral parenchymogram.

\section{Discussion}

Giant serpentine aneurysms are very rare and their etiology is still unclear, though some researchers have proposed that GSAs may arise due to repeated dissection with intramural thrombus formation. ${ }^{5}$ Patients with GSA often manifest signs of mass effect, and present intracranial mass rather than intracranial hemorrhage. ${ }^{4}$ The characteristic set of symptoms depend on the location of the aneurysm. Fifty percent of GSAs occurring in the region of the middle cerebral artery (MCA) can contribute to the predominant presenting symptoms, such as headache, nausea and vomiting, hemiparesis, dysphasia/aphasia, and seizure. Other symptoms often described in the literature include visual disturbance, cranial nerve palsy, papilledema, mental deterioration or depression, dysesthesia, and vertigo. ${ }^{2,4}$ Findings from literature suggest that only approximately $28 \%$ of patients present with subarachnoid hemorrhage ( $\mathrm{SAH}$ ) along with the typical associated headache., ${ }^{3,6}$

GSAs form a subcategory of intracranial giant aneurysms and possess certain distinguishing anatomic and radiographic features. The vascular channels may be central or eccentric 


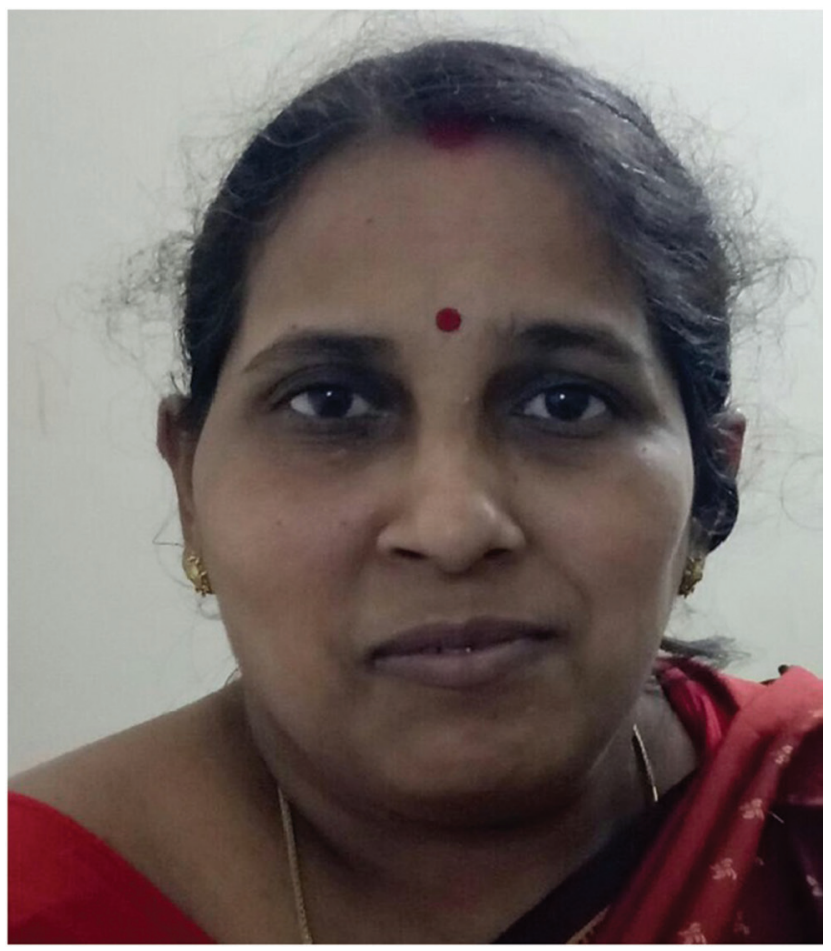

Fig. 9 Three-month postprocedure showing complete cure of ophthalmoplegia and proptosis.

within the aneurysm, with small branching channels that end blindly. Vascular flow is typically reported to be slow, which often reaches distal branches of the cerebral vasculature and eventually to vital or nonvital areas of the brain. Findings from literature describe these aneurysms as large globoid or pear-shaped masses with a 1.0 - to 4.0 -mm-thick fibrous wall that can possibly contain many small vessels similar to vasa vasorum. ${ }^{7.8}$ Their vascular channels do not appear to be residual lumina of the parent artery, but are typically observed to be intrathrombotic canals, which are not endothelialized and do not contain normal elastic lamina or media. The parent vessel is usually a branch of the middle or posterior cerebral artery, the distal vertebral artery at its junction with the basilar artery, or the supraclinoid internal carotid artery. ${ }^{4}$ In our case, the serpentine aneurysm involved the right internal carotid artery and supraclinoid segment in the right cavernous and had an irregular channel which continued into normal distal branches. In addition to GSA, the retroesophageal aortic arch and supra-aortic vascular anomaly added to the complexity of our case.

GSAs usually present with seizures or progressive neurologic deficits as a result of increasing intracranial mass effect and adjacent edema. Evidence from literature reports similar progression of a small fusiform aneurysm of branch of the middle cerebral artery into a GSA over a period of 5 years, thereby suggesting that the latter may have its origin in fusiform aneurysms that grow larger with time and undergo thrombosis and organization. ${ }^{7}$ Surgically removed GSAs have a thick fibrous wall that surrounds old laminated clots for the purpose of recanalization. The wall of the aneurysm contains neovessels, deposits of hemosiderin, and calcifications. ${ }^{9}$ Moreover, formation of a GSA after carotid ligation for treatment of a giant aneurysm has also been previously observed. ${ }^{8}$ In addition, a recent hypothesis hints at a possibility of continual growth of a dolichoectatic aneurysm leading to the formation of a supposed GSA. Our case here exhibits the growth of one such partially thrombosed dolichoectatic aneurysm to a serpiginous form. ${ }^{10}$ We may deduce that GSAs are not derived from saccular aneurysms, since they (1) do not originate from vessel bifurcations or vestigial vessels, (2) need an anatomic neck, and (3) have discrete entrance and exit sites in the vascular channel.

GSAs present characteristic radiologic findings. Plain-film radiographs can reveal pineal displacement caused by the mass, curvilinear calcification, and erosive change involving the skull base; while CT scans show an oval-shaped mass of mixed density. The nonenhanced scans present heterogeneous regions of increased attenuation indicating thrombus, and tubular regions of decreased attenuation indicating a patent vascular channel. When enhanced with the contrast, the serpiginous vascular channel is obvious. ${ }^{9,11}$ Although MR imaging findings of GSAs have not been comprehensively reported, they present a mass lesion with a heterogeneous signal representing various stages of degradation of hemoglobin and flow void regions. The aneurysm is evidently separated from normal parenchyma, possibly making the vascular channel visible on phase-contrast MR angiography. ${ }^{4}$ In our case, it was clearly visualized with very little clarity in few distal small branches.

Treatment of GSAs should be customized owing to their complex and varied hemodynamics and morphological features. Before the advent of revascularization surgery in the neurosurgical field, surgical treatment of GSAs contributed to significant (as high as $33 \%$ ) morbidity and mortality rates. ${ }^{12,13}$ The primary objective of the treatment of GSAs should be to (1) arrest their growth, (2) eliminate the mass effect, and (3) eliminate the abnormal vascular channel. An efficient way to achieve these is through direct and permanent occlusion of the parent artery including the aneurysm. This is achievable with endovascular means, that is, selective catheterization of the parent artery and occlusion of the vessel with detachable balloons, N-butyl cyanoacrylate, or Guglielmi detachable coils. ${ }^{4,14}$ However, occlusion of the parent artery through these endovascular means should be performed after cautious functional testing of the distal territory to assess any probable neurologic deficits that may follow. ${ }^{4}$

In our case, the retroesophageal aortic arch made it impossible to treat the GSA from the conventional, common femoral arterial route. Direct carotid puncture, brachial arterial access, agenesis of left ICA, and agenesis of VA posed technical challenge to treat the aneurysm. Fortunately, we could complete the procedure without any complication.

\section{Conclusion}

In conclusion, GSA is a fusiform, partially thrombosed aneurysm with tortuous vascular channels having separate outflow tracts to normal distal cerebral vessels. The CT, MR, or cerebral angiographic diagnostic modalities can provide 
critical information for the treatment of GSAs, such as endovascular occlusion of the parent vessel. The objective behind treating GSAs should be to stop the growth of the aneurysm, eliminate the mass effect, and destroy the abnormal vascular channel. This can be efficiently achieved through direct and permanent occlusion of the parent artery at the origin of the aneurysm. In a rare situation, a direct carotid puncture could be attempted when all other options are closed.

Conflict of Interest

None declared.

\section{References}

1 Segal HD, McLaurin RL. Giant serpentine aneurysm. Report of two cases. J Neurosurg 1977;46(1):115-120

2 Aletich VA, Debrun GM, Monsein LH, Nauta HJ, Spetzler RF. Giant serpentine aneurysms: a review and presentation of five cases. Am J Neuroradiol 1995;16(5):1061-1072

3 van Rooij WJ, Sluzewski M, Beute GN. Endovascular treatment of giant serpentine aneurysms. Am J Neuroradiol 2008;29(7):1418-1419

4 Park JS, Lee MS, Kim MS, Kim DJ, Park JW, Whang K. Giant serpentine intracranial aneurysm: a case report. Korean J Radiol 2001;2(3):179-182

5 Day AL, Gaposchkin CG, Yu CJ, Rivet DJ, Dacey RG Jr. Spontaneous fusiform middle cerebral artery aneurysms: characteristics and a proposed mechanism of formation. J Neurosurg 2003;99(2):228-240

6 Suzuki S, Takahashi T, Ohkuma H, Shimizu T, Fujita S. Management of giant serpentine aneurysms of the middle cerebral artery-review of literature and report of a case successfully treated by STA-MCA anastomosis only. Acta Neurochir (Wien) 1992;117(1-2):23-29

7 Tomasello F, Albanese V, Cioffi FA. Giant serpentine aneurysms: a separate entity. Surg Neurol 1979;12(5):429-432

8 Fodstad H, Liliequist B, Wirell S, Nilsson PE, Boquist L, Abdul-Rahman A. Giant serpentine intracranial aneurysm after carotid ligation. Case report. J Neurosurg 1978;49(6):903-909

9 Belec L, Cesaro P, Brugieres P, Gray F. Tumor-simulating giant serpentine aneurysm of the posterior cerebral artery. Surg Neurol 1988;29(3):210-215

10 Vishteh AG, Spetzler RF. Evolution of a dolichoectatic aneurysm into a giant serpentine aneurysm during long-term follow up. Case illustration. J Neurosurg 1999;91(2):346

11 Haddad GF, Haddad FS. Cerebral giant serpentine aneurysm: case report and review of the literature. Neurosurgery 1988;23(1):92-97

12 Albrecht S, Kahn HJ. Immunohistochemistry of intravascular papillary endothelial hyperplasia. J Cutan Pathol 1990;17(1):16-21

13 Chen TJ, Kuo T. Giant intracranial Masson's hemangioma. Report of a fatal case. Arch Pathol Lab Med 1984;108(7):555-556

14 Christiano LD, Gupta G, Prestigiacomo CJ, Gandhi CD. Giant serpentine aneurysms. Neurosurg Focus 2009;26(5):E5 